\title{
From Liveness to Promptness ${ }^{\star}$
}

\author{
Orna Kupferman ${ }^{1, \star \star}$, Nir Piterman ${ }^{2}$, and Moshe Y. Vardi ${ }^{3, \star \star \star}$ \\ ${ }^{1}$ Hebrew University \\ ${ }^{2}$ Ecole Polytechnique Fédéral de Lausanne (EPFL) \\ ${ }^{3}$ Rice University
}

\begin{abstract}
Liveness temporal properties state that something "good" eventually happens, e.g., every request is eventually granted. In Linear Temporal Logic (LTL), there is no a priori bound on the "wait time" for an eventuality to be fulfilled. That is, $F \theta$ asserts that $\theta$ holds eventually, but there is no bound on the time when $\theta$ will hold. This is troubling, as designers tend to interpret an eventuality $\mathbf{F} \theta$ as an abstraction of a bounded eventuality $\mathbf{F} \leq k \theta$, for an unknown $k$, and satisfaction of a liveness property is often not acceptable unless we can bound its wait time. We introduce here PROMPT-LTL, an extension of LTL with the prompt-eventually operator $\mathbf{F}_{\mathbf{p}}$. A system $S$ satisfies a PROMPT-LTL formula $\varphi$ if there is some bound $k$ on the wait time for all prompt-eventually subformulas of $\varphi$ in all computations of $S$. We study various problems related to PROMPT-LTL, including realizability, model checking, and assume-guarantee model checking, and show that they can be solved by techniques that are quite close to the standard techniques for LTL.
\end{abstract}

\section{Introduction}

Since the introduction of temporal logic into computer science [11], temporal logic, in its many different flavors, has been widely accepted as an appropriate formal framework for the description of on-going behavior of reactive systems [10]. Temporal properties are traditionally classified into safety and liveness properties [2]. Intuitively, safety properties assert that nothing bad will ever happen during the execution of the system, and liveness properties assert that something good will happen eventually. Temporal properties are interpreted with respect to systems that generate infinite computations. In satisfying liveness properties, there is no bound on the "wait time", namely the time that may elapse until an eventuality is fulfilled. For example, the LTL formula $\mathbf{F} \theta$ is satisfied at time $i$ if $\theta$ holds at some time $j \geq i$, but $j-i$ is not a priori bounded.

In many applications, it is important to bound the wait time. This has given rise to formalisms in which the eventually operator $\mathbf{F}$ is replaced by a bounded-eventually operator $\mathbf{F} \leq k$. The operator is parameterized by some $k \geq 0$, and it bounds the wait time to $k$ [49]. Since we assume that time is discrete, the operator $\mathbf{F} \leq k$ is simply a

\footnotetext{
${ }^{\star}$ Part of this work was done while the authors were visiting the Isaac Newton Institute for Mathematical Science, as part of a Special Programme on Logic and Algorithms. A full version can be downloaded from the authors' web sites.

** Supported in part by BSF grant 9800096 , and by a grant from Minerva.

$\star \star \star$ Supported in part by NSF grants CCR-9988322, CCR-0124077, CCR-0311326, CCF0613889, and ANI-0216467, by BSF grant 9800096, and by a Guggenheim Fellowship.
} 
syntactic sugar for an expression in which the next operator $\mathbf{X}$ is nested. Indeed, $\mathbf{F} \leq k \theta$ is just $\theta \vee \mathbf{X}(\theta \vee \mathbf{X}(\theta \vee k-4 \vee \mathbf{X} \theta))$.

A drawback of the above formalism is that the bound $k$ needs to be known in advance, which is not the case in many applications. For example, it may depend on the system, which may not yet be known, or it may change, if the system changes. In addition, the bound may be very large, causing the state-based description of the specification (e.g., an automaton for it) to be very large too. Thus, the common practice is to use liveness properties as an abstraction of such safety properties: one writes $\mathbf{F} \theta$ instead of $\mathbf{F} \leq k \theta$ for an unknown or a too large $k$.

For some temporal logics, the abstraction is sound, in the sense that if a system $S$ satisfies a liveness property $\psi$, then there is a bound $k$, which depends on $S$, such that $S$ also satisfies the formula obtained from $\psi$ by replacing all occurrences of $\mathbf{F}$ in $\psi$ by $\mathbf{F} \leq k$. For example, it is shown in [9] that in the case of CTL, taking $k$ to be the number of states in $S$ does it. Thus, if a state $s$ satisfies $A \mathbf{F} \theta$, then it also satisfies $A \mathbf{F} \leq k \theta$, for $k=|S|$, and similarly for $E \mathbf{F} \theta$. Intuitively, since $\theta$ is a state formula, a wait time that is greater than $|S|$ indicates that the wait time may also be infinite (by looping in a cycle that ought to be taken during the wait time), and may also be shortened to at most $|S|$ (by skipping such cycles).

So the abstraction of safety properties by liveness properties is sound for CTL. Is it sound also for the linear temporal logic LTL? Consider the system $\mathcal{S}$ described in Figure 1 below. While $\mathrm{S}$ satisfies the LTL formula $\mathbf{F G} q$, there is no $k \geq 0$ such that $\mathcal{S}$ satisfies $\mathbf{F} \leq k \mathbf{G} q$. To see this, note that for each $k \geq 0$, the computation that first loops in the first state for $k$ times and only then continues to the second state, satisfies the eventuality $\mathbf{G} q$ with wait time $k+1$.

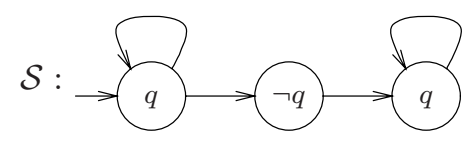

Fig. 1. $\mathcal{S}$ satisfies $\mathbf{F G} q$ but does not satisfy $\mathbf{F}^{\leq k} \mathbf{G} q$, for all $k \geq 0$

It follows that the abstraction of safety properties by liveness properties is not sound in the linear-time approach (which is more popular with users, cf. [7]). This is troubling, as designers tend to interpret eventualities as bounded eventualities, and satisfaction of a liveness property is often not acceptable unless we can bound its wait time 1

In this work we introduce and study an extension of LTL that addresses the above problem. In addition to the usual temporal operators of LTL, our logic, PROMPT-LTL, has a new temporal operator that is used for specifying eventualities with a bounded wait time. We term the operator prompt eventually and denote it by $\mathbf{F}_{\mathbf{p}}$. Let us define the semantics of PROMPT-LTL formally. For a PROMPT-LTL formula $\psi$ and a bound $k \geq 0$, let $\psi^{k}$ be the LTL formula obtained from $\psi$ by replacing all occurrences of $\mathbf{F}_{\mathbf{p}}$ by $\mathbf{F} \leq k$. Then, a system $S$ satisfies $\psi$ iff there is $k \geq 0$ such that $S$ satisfies $\psi^{k}$.

\footnotetext{
${ }^{1}$ Note that the reduction of liveness to safety as described in [3] is performed by squaring the state space rather than trying to bound the wait time of eventualities. Thus, it is not related to the discussion in this paper.
} 
Note that while the syntax of PROMPT-LTL is very similar to that of LTL, its semantics is defined with respect to an entire system, and not with respect to computations. For example, while each computation $\pi$ in the system $\mathcal{S}$ from Figure 1 has a bound $k_{\pi} \geq 0$ such that $\mathbf{G} q$ is satisfied in $\pi$ with wait time $k_{\pi}$, there is no $k \geq 0$ that bounds the wait time of all computations. It follows that, unlike linear temporal logics, we cannot characterize a PROMPT-LTL formula $\psi$ over a set $A P$ of atomic propositions by a set of computations $L_{\psi} \subseteq\left(2^{A P}\right)^{\omega}$ such that a system $S$ satisfies $\psi$ iff the language of $S$ is contained in $L_{\psi}$. On the other hand, unlike branching temporal logics, if two systems agree on their languages, then they agree also on the satisfaction of all PROMPTLTL formulas. Thus, PROMPT-LTL intermediates between the linear and branching approaches: as in the linear approach, the specification refers to the set of computations of the system rather than its computation tree; as in the branching approach, we cannot consider these computations individually.

As further motivation to a prompt eventuality operator, consider the formula $\mathbf{F} a \rightarrow$ $\mathbf{F} b$. A system may satisfy $\mathbf{G} \neg a \vee \mathbf{F} b$ but have no bound on the wait time to the satisfaction of the eventuality. When a user checks $\mathbf{F} a \rightarrow \mathbf{F} b$, it is quite likely that what he has in mind is $\mathbf{G} \neg a \vee \mathbf{F}_{\mathbf{p}} b$. The user may not know a bound $k$ such that $\mathbf{G} \neg a \vee \mathbf{X} \leq k b$ should be checked. It is also possible that what the user has in mind is "assume $\mathbf{F a}$; assert $\mathbf{F} b$ ", where the bound for $b$ ought to depend on the bound for $a$. Our semantics distinguishes these three different understandings of $\mathbf{F} a \rightarrow \mathbf{F} b$.

We study the basic problems of PROMPT-LTL. Consider a PROMPT-LTL formula $\psi$ over $A P$. The set $A P$ may be partitioned to sets $I$ and $O$ of input and output signals. Consider also a system $S$. We study the following problems: realizability (is there a strategy $f:\left(2^{I}\right)^{*} \rightarrow 2^{O}$ such that all the computations generated by $f$ satisfy $\psi$ ?), model checking (does $S$ satisfy $\psi$ ?), and assume-guarantee model checking (given an additional PROMPT-LTL formula $\varphi$, is it the case that for all systems $S^{\prime}$, if $S \| S^{\prime}$ satisfies $\varphi$, then $S \| S^{\prime}$ also satisfies $\psi$ ?). Since a system that satisfies a PROMPT-LTL formula may consist of a single regular computation, the satisfiability problem for prompt-LTL can be easily reduced to LTL satisfiability (simply replace all occurrences of $\mathbf{F}_{\mathbf{p}}$ by $\mathbf{F}$ ). For the other problems, similar reductions do not work, and we have to develop a new technique in order to solve them. Let us describe our technique briefly.

Consider a prompt-LTL formula $\psi$ over $A P$. Let $p$ be an atomic proposition not in $A P$. Think about $p$ as a description of one of two colors, say green ( $p$ holds) and red ( $p$ does not hold). Each computation of the system can be partitioned to blocks such that states of the same block agree on their color. We show that a system $S$ satisfies a PROMPT-LTL formula $\psi$ iff there is some bound $k \geq 0$ such that we can color each computation $\pi$ of $S$ so that the induced blocks are of length $k$, and whenever a suffix of $\pi$ has to satisfy an eventuality, the eventuality is fulfilled within two blocks. Indeed, the latter condition holds iff all eventualities have wait time at most $2 k$.

The key idea behind our technique is that rather than searching for a bound $k$ for the prompt eventualities, which can be quite large, it is enough to make sure that there is a coloring in which all blocks are of a (not necessarily bounded) finite length, and then use some regularity argument in order to conclude that the size of the blocks could actually be bounded. Forcing the blocks to be of a finite length can be done by requiring the colors to alternate infinitely often. As for regularity, in the case of realizability, 
regularity follows from the finite-model property of tree automata. In the case of (assume-guarantee) model checking, regularity follows from the finiteness of the system.

The complexities that follow from our algorithms are encouraging: reasoning about PROMPT-LTL is not harder than reasoning about LTL: realizability is 2EXPTIMEcomplete, and model checking and assume-guarantee model checking are PSPACEcomplete. For LTL, many heuristics have been studied and applied. Some of them are immediately applicable for PROMPT-LTL (c.f., optimal translations of formulas to automata), and some should be extended to the prompt setting (e.g., bad-cycle detection algorithms). We also study some theoretical aspects of PROMPT-LTL, such as the ability to translate PROMPT-LTL formulas to branching-temporal logics (a translation to the $\mu$-calculus is always possible, but may involve a significant blow up), and the ability to determine whether a PROMPT-LTL formula has an equivalent LTL formula (PSPACEcomplete).

\section{Prompt Linear Temporal Logic}

The logic PROMPT-LTL extends LTL [11] by a prompt-eventually operator $\mathbf{F}_{\mathbf{p}}$. The syntax of PROMPT-LTL formulas (in negation normal form) is given by the grammar below, for a set $A P$ of atomic propositions: $\varphi:=A P|\neg A P| \varphi \vee \varphi \mid \varphi \wedge$ $\varphi|\mathbf{X} \varphi| \mathbf{F}_{\mathbf{p}} \varphi|\varphi \mathbf{U} \varphi| \varphi \mathbf{R} \varphi$. The semantics of a PROMPT-LTL formula is defined with respect to an infinite word $w=w_{0}, w_{1}, \ldots$ over the alphabet $2^{A P}$, a position $i \geq 0$ in $w$, and a bound $k \geq 0$. We use $(w, k, i) \models \varphi$ to indicate that $\varphi$ holds in location $i$ of $w$ with bound $k$. The relation $\models$ is defined by induction on the structure of $\varphi$ as follows.

- For propositions, Boolean connectives, and LTL temporal operators, the definition is independent of $k$ and coincides with the one for LTL2

- $(w, i, k) \models \mathbf{F}_{\mathbf{p}} \varphi$ iff there exists $j$ such that $i \leq j \leq i+k$ and $(w, j, k) \models \varphi$.

We use $\mathbf{F} \theta$ and $\mathbf{G} \theta$ to abbreviate true $\mathbf{U} \theta$ and false $\mathbf{R} \theta$, respectively. Note that the negation of $\mathbf{F}_{\mathbf{p}}$ is not expressible in PROMPT-LTL, thus the logic is not closed under negation. Given a PROMPT-LTL formula $\varphi$, let $\operatorname{live}(\varphi)$ be the LTL formula obtained from $\varphi$ by replacing every prompt-eventually operator $\mathbf{F}_{\mathbf{p}}$ by a standard eventually operator $\mathbf{F}$.

A (labeled) transition system is $\mathcal{S}=\left\langle A P, S, \rho, s_{0}, L\right\rangle$, where $A P$ is a finite set of atomic propositions, $S$ is a finite set of states, $\rho \subseteq S \times S$ is a total transition relation, $s_{0} \in S_{0}$ is an initial state, and $L: S \rightarrow 2^{A P}$ maps each state $s$ to the set of propositions that hold in $s$. When $\rho\left(s, s^{\prime}\right)$, we say that $s^{\prime}$ is a successor of $s$, and $s$ is a predecessor of $s^{\prime}$. A computation of $\mathcal{S}$ is an infinite sequence of states $\pi=s_{0}, s_{1}, \ldots \in S^{\omega}$ such that for all $i \geq 0$, we have $\rho\left(s_{i}, s_{i+1}\right)$. The computation $\pi$ induces the trace $L(\pi)=$ $L\left(s_{0}\right) \cdot L\left(s_{1}\right) \cdots$.

Given a system $\mathcal{S}$ and a PROMPT-LTL formula $\varphi$ over $A P$, we say that $\mathcal{S}$ satisfies $\varphi$, denoted $\mathcal{S}=\varphi$, if there exists some $k \geq 0$ such that for all traces $w$ of $\mathcal{S}$, we have $(w, 0, k) \models \varphi$. We then say that $\mathcal{S}$ satisfies $\varphi$ with bound $k$. Note that when $\mathcal{S} \forall \varphi$, then for every $k \geq 0$, there exists a trace $w$ such that $(w, 0, k) \not \models \varphi$.

${ }^{2}$ Recall that in LTL we have that $\pi, i \models \theta R \psi$ iff for all $j \geq i$, if $\pi, j \forall \psi \psi$, then for some $k$, $i \leq k<j$, we have $\pi, k \models \theta$. 
In [1], Alur et al. study an extension of LTL in which the temporal operators $\mathbf{F}$ and $\mathbf{G}$ are replaced by the operators $\mathbf{F}_{\leq x}, \mathbf{F}_{>y}, \mathbf{G}_{\leq x}$, and $\mathbf{G}_{>y}$, for variables $x$ and $y$ (the same variable may be used in different operators, but, to ensure decidability, the same variable cannot participate in both a lower and an upper bound). Given a system $\mathcal{S}$ and a formula in their logic, one can ask whether there is an assignment to the variables for which the system satisfies the formula, with the expected interpretation of the bounded operators 3 Our logic can be viewed as a special case of the logic studied in [1], in which only eventualities are parameterized, and only with upper bounds. The algorithms suggested by Alur et al. are rather involved. By giving up the operators $\mathbf{F}_{>y}, \mathbf{G}_{\leq x}$, and $\mathbf{G}_{>y}$, whose usefulness is debatable, we get a much simpler model-checking algorithm, which is also similar to the classical LTL model-checking algorithm. We are also able to a solve the realizability and the assume-guarantee model checking problems.

The Alternating-Color Technique. We now describe the key idea of our technique for reasoning about PROMPT-LTL formulas. Let $p$ be an atomic proposition not in $A P$. We think about $p$ as a description of one of two colors, say green ( $p$ holds) and red ( $p$ does not hold). Each computation of the system can be partitioned to blocks such that states of the same block agree on their color. Our technique is based on the idea that bounding the wait time of prompt eventualities can be reduced to forcing all blocks to be of a bounded length, and forcing all eventualities to be fulfilled within two blocks, We now make this intuition formal.

Consider a word $w=\sigma_{0}, \sigma_{1}, \ldots \in\left(2^{A P}\right)^{\omega}$. Let $p$ be a proposition not in $A P$. A p-coloring of $w$ is a word $w^{\prime}=\sigma_{0}^{\prime}, \sigma_{1}^{\prime}, \ldots \in\left(2^{A P \cup\{p\}}\right)^{\omega}$ such that $w^{\prime}$ agrees with $w$ on the propositions in $A P$; i.e., for all $i \geq 0$, we have $\sigma_{i}^{\prime} \cap A P=\sigma_{i}$. We refer to the assignment to $p$ as the color of location $i$ and say that $i$ is green if $p \in \sigma_{i}^{\prime}$ and is red if $p \notin \sigma_{i}^{\prime}$. We say that $p$ changes at $i$ if either $i=0$ or the colors of $i-1$ and $i$ are different (that is, $p \in \sigma_{i-1}^{\prime}$ iff $p \notin \sigma_{i}^{\prime}$ ). We then call $i$ a $p$-change point. A subword $\sigma_{i}^{\prime}, \ldots, \sigma_{i^{\prime}}^{\prime}$ is a $p$-block if all positions in the subword have the same color, and $i$ and $i^{\prime}+1$ are $p$-change points. We then say that $i$ and $i^{\prime}+1$ are adjacent $p$-change points. For $k \geq 0$, we say that $w^{\prime}$ is $k$-spaced, $k$-bounded, and $k$-tight (with respect to $p$ ) if $w^{\prime}$ has infinitely many blocks, and all the blocks are of length at least $k$, at most $k$, and exactly $k$, respectively.

Consider the formula alt $_{p}=\mathbf{G F} p \wedge \mathbf{G F} \neg p$. It requires that the proposition $p$ alternates infinitely often. Given a PROMPT-LTL formula $\varphi$, let $\operatorname{rel}_{p}(\varphi)$ denote the formula obtained from $\varphi$ by (recursively) replacing each subformula of the form $\mathbf{F}_{\mathbf{p}} \psi$ by the LTL formula $(p \rightarrow(p \mathbf{U}(\neg p \mathbf{U} \psi))) \wedge(\neg p \rightarrow(\neg p \mathbf{U}(p \mathbf{U} \psi)))$. Note that the definition is recursive, thus $\operatorname{rel}_{p}(\varphi)$ may be exponentially larger than $\varphi$. The number of subformulas of $\operatorname{rel}_{p}(\varphi)$, however, is linear in the number of subformulas of $\varphi$, and it is this number that plays a role in the complexity analysis (equivalently, the size of the DAG-presentation of $\operatorname{rel}_{p}(\varphi)$ is linear in the size of the DAG presentation of $\varphi$ ). For a PROMPT-LTL formula $\varphi$, we define $c(\varphi)=\operatorname{alt}_{p} \wedge \operatorname{rel}_{p}(\varphi)$. Thus, $c(\varphi)$ forces the computation to be partitioned into infinitely many blocks, and requires each prompt

${ }^{3}$ The work in [1] studies many more aspects of the logic, like the problem of deciding whether the formula is satisfied with all assignments, the problem of finding an optimal assignment, and other decidability issues. 
eventuality to be satisfied in the current or next block or in the position immediately after the next block (within two blocks, for short),

Lemma 1. Consider a PROMPT-LTL formula $\varphi$, a word $w$, and $a$ bound $k \geq 0$.

1. If $(w, 0, k) \models \varphi$, then $\left(w^{\prime}, 0\right) \models c(\varphi)$, for every $k$-spaced $p$-coloring $w^{\prime}$ of $w$.

2. If $w^{\prime}$ is a $k$-bounded $p$-coloring of $w$ such that $\left(w^{\prime}, 0\right) \models c(\varphi)$, then $(w, 0,2 k) \models \varphi$.

The alternating-color technique sets the basis to reasoning about a PROMPT-LTL formula $\varphi$ by reasoning about the LTL formula $c(\varphi)$. The formula $c(\varphi)$, however, does not require the blocks in the colored computation to be of a bounded length. Indeed, the conjunct $a l t t_{p}$ only forces the colors to be finite, and it does not prevent, say, a $p$-coloring in which each block is longer than its predecessor block, and which is not $k$-bounded, for all $k \geq 0$. Thus, the challenge of forcing the $p$-coloring to be $k$-bounded for some $k$ remains, and we have to address it in each of the decision procedures described in the following sections.

\section{Realizability}

Given an LTL formula $\psi$ over the sets $I$ and $O$ of input and output signals, the realizability problem for $\psi$ is to decide whether there is a strategy $f:\left(2^{I}\right)^{*} \rightarrow 2^{O}$ such that all the computations generated by $f$ satisfy $\psi[13]$. Formally, a computation $w \in\left(2^{I \cup O}\right)^{\omega}$ is generated by $f$ if $w=\left(i_{0} \cup o_{0}\right),\left(i_{1} \cup o_{1}\right),\left(i_{2} \cup o_{2}\right), \ldots$ and for all $j \geq 0$, we have $o_{j}=f\left(i_{0} \cdot i_{1} \cdots i_{j}\right)$. Thus, the interaction is initiated by the environment that generates $i_{0}$, and the first state in the computation is labeled $i_{0} \cup f\left(i_{0}\right)$. Then, the environment generates $i_{1}$, and the second state in the computation is $i_{1} \cup f\left(i_{0} \cdot i_{1}\right)$, and so on. It is known that if some strategy that realizes $\psi$ exists, then there also exists a regular strategy (i.e, a strategy generated by a finite-state transducer) that realizes $\psi$ [6]. Formally, a transducer is $\mathcal{D}=\left\langle I, O, Q, \eta, q_{0}, L\right\rangle$, where $I$ and $O$ are the sets of input and output signals, $Q$ is a finite set of states, $\eta: Q \times 2^{I} \rightarrow Q$ is a deterministic transition function, $q_{0} \in Q$ is an initial state, and $L: Q \rightarrow 2^{O}$ maps each state to a set of output signals. The transducer $\mathcal{D}$ generates $f$ in the sense that for every $\tau \in\left(2^{I}\right)^{*}$, we have $f(\tau)=L(\eta(\tau))$, with the usual extension of $\eta$ to words over $2^{I}$.

We first show that PROMPT-LTL realizability of a formula $\varphi$ cannot be simply reduced to the realizability of live $(\varphi)$. Thus, we describe a formula $\varphi$ such that live $(\varphi)$ is realizable, but for every strategy $f$ that realizes $\varphi$ and for every candidate bound $k \geq 0$, there is a computation $w$ generated by $f$ such that $(w, 0, k) \not \models \varphi$. Let $I=\{i\}$ and $O=\{o\}$. We define $\varphi=o \wedge(\mathbf{G}(i \rightarrow o)) \wedge((\mathbf{X} \neg o) \mathbf{R} i) \wedge\left(\mathbf{F}_{\mathbf{p}} \mathbf{G} o\right)$.

Thus, a computation satisfies $\varphi$ if $o$ holds in the present and whenever $i$ holds, whenever $i$ does not hold in some position, then $o$ does not hold in this position or in an earlier one, and the computation prompt-eventually reaches a position from which $o$ holds everywhere. It is not hard to see that $\operatorname{live}(\varphi)$ is realizable. Indeed, the strategy that sets $o$ to true everywhere except in the first time that $i$ is false realizes live $(\varphi)$. On the other hand, $\varphi$ is not realizable. To see this, note that the position in which the input $i$ is set to false can be delayed arbitrarily by the environment, forcing a delay also in the fulfillment of the $\mathbf{G} o$ eventuality. Thus, for every candidate bound $k \geq 0$, the 
input sequence in which $i$ is false at the $(k+1)$-th position cannot be extended to a computation that satisfies $\mathbf{F}_{\mathbf{p}} \mathbf{G} o$ with bound $k$.

The good news is that while realizability of $\varphi$ cannot be reduced to the realizability of live $(\varphi)$, it can be reduced to the realizability of $c(\varphi)$. Intuitively, it follows from the fact that in a regular strategy, the fact that all blocks are of a finite length does imply that they are also of a bounded length. Formally, we have the following.

Theorem 1. A PROMPT-LTL formula $\varphi$ over input signals $I$ and output signals $O$ is realizable iff the LTL formula $c(\varphi)$ over input signals I and output signals $O \cup\{p\}$ is realizable.

Since LTL realizability is 2EXPTIME-complete and every LTL formula is also a PROMPT-LTL formula, we can conclude:

Theorem 2. The problem of prompt realizability is 2EXPTIME-complete in the size of the formula.

As demonstrated above, the alternating-color technique is very powerful in the case of realizability. Indeed, the challenge of forcing the $p$-coloring to be $k$-bounded for some $k$ is taken care of by the regularity of the strategy. We now proceed to the model-checking problem, where a reduction to $c(\varphi)$ is not sufficiently strong.

\section{Model Checking}

In this section we describe an algorithm for solving the model-checking problem for PROMPT-LTL. An alternative algorithm is described for the richer parameterized linear temporal logic in [1]. Our algorithm is much simpler, and it deviates from the standard LTL model-checking algorithm only slightly. In addition, as we show in Section 6 the idea behind our algorithm can be applied also in order to solve assume-guarantee model checking, which is not known to be the case with the algorithm in [1]. Our algorithm is based on the automata-theoretic approach to LTL model-checking, and we first need some definitions.

A nondeterministic Büchi word automaton (NBW for short) is $\mathcal{A}=\left\langle\Sigma, S, \delta, s_{0}, \alpha\right\rangle$, where $\Sigma$ is a finite alphabet, $S$ is a finite set of states, $\delta: S \times \Sigma \rightarrow 2^{S}$ is a transition function, $s_{0} \in S$ is an initial state, and $\alpha \subseteq S$ is a Büchi acceptance condition. A run of $\mathcal{A}$ on a word $w=w_{0} \cdot w_{1} \cdots$ is an infinite sequence of states $s_{0}, s_{1}, \ldots$ such that $s_{0}$ is the initial state and for all $j \geq 0$, we have $s_{j+1} \in \delta\left(s_{j}, w_{j}\right)$. For a run $r=s_{0}, s_{1}, \ldots$, let $\inf (r)=\left\{s \in S \mid s=s_{i}\right.$ for infinitely many $i$ 's $\}$ be the set of all states occurring infinitely often in the run. A run is accepting if $\inf (r) \cap \alpha \neq \emptyset$. That is, the run visits infinitely many states from $\alpha$. A word $w$ is accepted by $\mathcal{A}$ if there exists some accepting run of $\mathcal{A}$ over $w$. The language of $\mathcal{A}$, is the set of words accepted by $\mathcal{A}$.

Theorem 3. [17] For every LTL formula $\varphi$ over $A P$ there exists an $N B W \mathcal{A}_{\varphi}$ over the alphabet $2^{A P}$ such that $\mathcal{A}_{\varphi}$ accepts exactly all words that satisfy $\varphi$. The number of states of $\mathcal{A}_{\varphi}$ is at most exponential in the number of subformulas of $\varphi$.

In order to check whether a system $\mathcal{S}$ satisfies an LTL formula $\varphi$, one takes the product of $\mathcal{S}$ with the NBW $\mathcal{A}_{\neg \varphi}$ and tests the product for non-emptiness [16]. Indeed, a path in 
this product witnesses a computation of $\mathcal{S}$ that does not satisfy $\varphi$. As discussed in Section 1 in the case of PROMPT-LTL we cannot translate formulas to languages. Moreover, we also cannot simply apply the alternating-color technique: even if we check the nonemptiness of the product of the system (an augmentation of it in which the proposition $p$ behaves nondeterministically, thus all $p$-colorings are possible) with the automaton for alt $_{p} \wedge \neg \operatorname{rel}_{p}(\varphi)$, a path in this product only implies that for some bound $k \geq 0$, the formula $\varphi$ is not satisfied in $\mathcal{S}$ with bound $k$. For proving that $\mathcal{S}$ does not satisfy $\varphi$ we have to prove something stronger, namely, that $\varphi$ is not satisfied in $\mathcal{S}$ with bound $k$, for all bounds $k \geq 0$. For that, we do take the product of the system with the automaton for alt $_{p} \wedge \neg \operatorname{rel}_{p}(\varphi)$, but add a twist to the nonemptiness check: we search for a path in the product in which each $p$-block contains at least one state that repeats. Such a state indicates that for all bounds $k \geq 0$, the $p$-block can be pumped to a $p$-block of length greater than $k$, implying that $\varphi$ cannot be satisfied in $\mathcal{S}$ with bound $k$. We now formalize this intuition.

A colored Büchi graph is a tuple $G=\left\langle\{p\}, V, E, v_{0}, L, \alpha\right\rangle$, where $p$ is a proposition, $V$ is a set of vertices, $E \subseteq V \times V$ is a set of edges, $v_{0} \in V$ is an initial vertex, $L: V \rightarrow 2^{\{p\}}$ describes the color of each vertex, and $\alpha \subseteq V$ is a set of accepting states. A path $\pi=v_{0}, v_{1}, v_{2}, \ldots$ of $G$ is pumpable if all its $p$-blocks have at least one state that repeats. Formally, if $i$ and $i^{\prime}$ are adjacent $p$-change points, then there are positions $j$ and $j^{\prime}$ such that $i \leq j<j^{\prime}<i^{\prime}$ and $v_{j}=v_{j^{\prime}}$. Also, $\pi$ is fair if it visits $\alpha$ infinitely often. The pumpable nonemptiness problem is to decide, given $G$, whether is has a pumpable fair path.

Let $\bar{c}(\varphi)=\operatorname{alt}_{p} \wedge \neg \operatorname{rel}_{p}(\varphi)$. That is, we relativize the satisfaction of $\mathbf{F}_{\mathbf{p}}$ to the new proposition $p$, negate the resulting formula, and require the proposition $p$ to alternate infinitely often. Let $\mathcal{A}_{\bar{c}(\varphi)}=\left\langle 2^{A P \cup\{p\}}, Q, \delta, q_{0}, \alpha\right\rangle$ be the NBW for $\bar{c}(\varphi)$ per Theorem 3 Consider a system $\mathcal{S}=\left\langle A P, S, \rho, s_{0}, L\right\rangle$. We now define the product of $\mathcal{S}$ with $\mathcal{A}_{\bar{c}(\varphi)}$ by means of a colored Büchi graph. Note that $\mathcal{S}$ does not refer to the proposition $p$, and we duplicate its state space in order to have in the product all possible $p$-colorings of computations in $\mathcal{S}$. Thus, the product is $\mathcal{P}=\left\langle\{p\}, S \times\{\{p\}, \emptyset\} \times Q, M,\left\langle s_{0},\{p\}, q_{0}\right\rangle\right.$, $L, S \times\{\{p\}, \emptyset\} \times \alpha\rangle$, where $M\left(\langle s, c, q\rangle,\left\langle s^{\prime}, c^{\prime}, q^{\prime}\right\rangle\right)$ iff $\rho\left(s, s^{\prime}\right)$ and $q^{\prime} \in \delta(q, L(s) \cup c)$, and $L(\langle s, c, q\rangle)=c$.

It is not hard to see that a path $\pi=\left\langle s_{0}, c_{0}, q_{0}\right\rangle,\left\langle s_{1}, c_{1}, q_{1}\right\rangle,\left\langle s_{2}, c_{2}, q_{2}\right\rangle, \ldots$ in $\mathcal{P}$ corresponds to a computation $s_{0}, s_{1}, s_{2}, \ldots$ of $\mathcal{S}$, a $p$-coloring $L\left(s_{0}\right) \cup c_{0}, L\left(s_{1}\right) \cup$ $c_{1}, L\left(s_{2}\right) \cup c_{2}, \ldots$ of the trace that the computation induces, and a run $q_{0}, q_{1}, q_{2}, \ldots$ of $\mathcal{A}_{\bar{c}(\varphi)}$ on this $p$-coloring.

Theorem 4. The system $\mathcal{S}$ does not satisfy $\varphi$ iff the product of $\mathcal{S}$ and $\mathcal{A}_{\bar{c}(\varphi)}$ is pumpable nonempty.

In Section [5] we study the problem of deciding whether a colored Büchi graph is pumpable-nonempty, and prove that it is in NLOGSPACE and can also be solved in linear time. This, together with Theorems 3 and 4 imply the upper bound in the following theorem. The lower bound follows from the known lower bound for LTL.

Theorem 5. The model-checking problem for PROMPT-LTL is PSPACE-complete and can be solved in time exponential in the length of the formula and linear in the size of the system. 
Note that while the pumpable nonemptiness problem to which PROMPT-LTL modelchecking is reduced is a variant of the nonemptiness problem to which LTL model checking is reduced, the construction of the product is almost the same. In particular, the extensive work on optimal compilation of LTL formulas to NBW (see survey in [15]), is applicable to our solution too.

Remark 6. The model-checking algorithm of the parametric linear temporal logic of [1] is based on the observation that if a PROMPT-LTL formula $\varphi$ is satisfied in a system $\mathcal{S}$, then it is satisfied with bound $k$, for some $k$ that is exponential in $\varphi$ and polynomial in $\mathcal{S}$. One cannot hope to improve this bound. Indeed, for every $n \geq 1$, we can define a PROMPT-LTL formula $\psi_{n}$ of size linear in $n$ such that a systems satisfies $\psi_{n}$ iff in all its computations, the atomic proposition $q$ corresponds to an $n$-bit counter, and the value of the counter promptly eventually reaches $2^{n}-1$. Clearly, $\psi_{n}$ is promptly satisfied, but the minimal bound $k$ with which $\psi_{n}$ is satisfied with bound $k$ (in some system) is exponential in $n$.

The algorithm in [1] can also be used in order to find the minimal bound. It is an open question whether the minimal bound can be found using our simplified algorithm.

\section{Algorithms for Colored Büchi Graphs}

In Section 4 we reduced model-checking for PROMPT-LTL to pumpable nonemptiness problems for colored Büchi graphs. In this section we solve this problems, and provide space and time bounds.

Theorem 7. The pumpable nonemptiness problem for colored Büchi graphs is NLOGSPACE-complete and can be solved in linear time.

Proof: Let $G=\left\langle\{p\}, V, E, v_{0}, L, \alpha\right\rangle$. We start with the space complexity. Essentially, as with standard Büchi nonemptiness, the pumpable nonemptiness problem can be solved by a sequence of reachability tests. In addition to reaching a vertex $v$ in $\alpha$ that is reachable from itself, the algorithm should make sure that the paths from $v_{0}$ to $v$ and from $v$ to itself are pumpable. Thus, in each $p$-block, the algorithm should guess a repeated vertex (and check that it indeed repeats). Also, an easy reduction from reachability shows hardness in NLOGSPACE.

We now move to the time complexity. For standard Büchi nonemptiness, one looks for a reachable nontrivial strongly connected component that intersects $\alpha$. In the colored case, we should further check that each $p$-block in the path can be pumped. We do this by making sure that every green $p$-block contains at least one vertex that belongs to a nontrivial strongly connected component in the graph of the green vertices, and similarly for the red $p$-blocks.

Consider the graph $G_{g}=\left\langle V_{g}, E_{g}\right\rangle$ obtained from $G$ by restricting attention to green vertices. Thus, $V_{g}=\{v \in V \mid L(v)=\{p\}\}$ and $E_{g}=E \cap\left(V_{g} \times V_{g}\right)$. The graph $G_{r}=$ $\left\langle V_{r}, E_{r}\right\rangle$ is defined similarly. We can find the maximal strongly connected components (MSCC) of $G_{g}$ and $G_{r}$ in linear time [14] (note we are interested also in MSCCs that are not reachable from $v_{0}$ in $G_{g}$ and $G_{r}$ ). Let $S_{g} \subseteq V_{g}$ and $S_{r} \subseteq V_{r}$ denote the union of all non-trivial MSCCs in $G_{g}$ and $G_{r}$, respectively. 
Let $\operatorname{back}_{g}\left(S_{g}\right)$ be the vertices that can reach some vertex in $S_{g}$, and let $e$-back $k_{g}\left(S_{g}\right)$ be the edges that are used to reach these vertices. We tag the vertices in $\operatorname{back}_{g}\left(S_{g}\right) \backslash S_{g}$ by the tag B. Formally, we define back $_{0}^{g}\left(S_{g}\right)=S_{g}$ and back $_{i+1}^{g}\left(S_{g}\right)=\left\{v \in V_{g} \mid \exists v^{\prime} \in\right.$ $\operatorname{back}_{i}^{g}\left(S_{g}\right)$ and $\left.\left(v, v^{\prime}\right) \in E\right\}$. Then, $\operatorname{back}_{g}\left(S_{g}\right)=S_{g} \cup\left(\bigcup_{i \geq 1} \operatorname{back}_{i}^{g}\left(S_{g}\right)\right) \times\{\mathrm{B}\}$. For a vertex $u \in \operatorname{back}_{g}\left(S_{g}\right)$, let $\operatorname{ver}(u)$ be the vertex in $V$ that induces $u$; that is, the vertex obtained from $u$ by ignoring its tag, if exists. Then, $e$-back $k_{g}\left(S_{g}\right)=\left\{\left\langle u, u^{\prime}\right\rangle\right.$ : $E\left(\operatorname{ver}(u), \operatorname{ver}\left(u^{\prime}\right)\right)$ and there is $i \geq 0$ such that $u \in \operatorname{back}_{i+1}^{g}\left(S_{g}\right)$ and $\left.u^{\prime} \in \operatorname{back}_{i}^{g}\left(S_{g}\right)\right\}$. In a similar way, we define forward ${ }_{g}\left(S_{g}\right)$ to be the set of vertices that are reachable from some vertex in $S_{g}$ (with vertices not in $S_{g}$ tagged with F) and define $e$-forward ${ }_{g}\left(S_{g}\right)$ to be the edges that are used to reach these vertices. The sets back $_{r}, e$-back , forward $_{r}$, and $e$-forward $r$ are defined similarly. Another type of edges we need are edges between $p$-blocks. Let $E_{g \rightarrow r}=\left\{\left\langle u, u^{\prime}\right\rangle: E\left(\operatorname{ver}(u), \operatorname{ver}\left(u^{\prime}\right)\right), u \in\right.$ forward $_{g}\left(S_{g}\right)$, and $u^{\prime} \in$ $\left.\operatorname{back}_{r}\left(S_{r}\right)\right\}$ be the set of edges along which the color changes from green to red, and let $E_{r \rightarrow g}$ be the set of edges along which the color changes from red to green.

Consider now the graph $G^{\prime}=\left\langle V^{\prime}, E^{\prime}\right\rangle$, where $V^{\prime}=\operatorname{back}_{g}\left(S_{g}\right) \cup$ forward $_{g}\left(S_{g}\right) \cup$

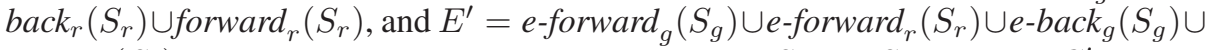
$e$-back ${ }_{r}\left(S_{r}\right) \cup E_{g \rightarrow r} \cup E_{r \rightarrow g}$. Note that the vertices in $S_{g}$ and $S_{r}$ appear in $G^{\prime}$ with no tag. Other vertices (these in $V_{g}$ that can reach an MSCC in $S_{g}$ along green vertices and can also be reached from a different MSCC in $S_{g}$ along green vertices, and similarly for $V_{r}$ ) may appear in $G^{\prime}$ with both tags, thus the number of vertices in $G^{\prime}$ is at most twice the number of vertices in $G$.

Intuitively, the graph $G^{\prime}$ contains exactly all the pumpable computations of $G$. Indeed, along each $p$-block, there must exists a vertex that belongs to an MSCC of the graph of the corresponding color. In the full version, we prove that $G$ is pumpable nonempty iff $G^{\prime}$ has some non-trivial MSCC that is reachable from $v_{0}$ (possibly tagged with B) and contains a vertex from $\alpha$.

We analyze the time it takes to construct $G^{\prime}$ and to check whether it has a non-trivial MSCC that intersects $\alpha$. Clearly, the MSCC decomposition of $G_{g}$ and $G_{r}$ can be done in linear time. The search for back and forward $_{g}$ is done by backward (resp. forward) propagation from $S_{g}$, during which the edges in $e$-back b $_{g}$ and $e$-forward fan $_{g}$ be marked. The case of back $_{r}$ and forward $r$ is similar. This stage can be completed in linear time as well. Finally, the MSCC decomposition of $G^{\prime}$ is completed again in linear time, thus the overall running time is linear.

We note than our algorithm is based in MSCC-decomposition. It is an open question whether a linear-time algorithm based on nested depth-first-search can be found (see discussion of these types of algorithms in [15]).

Remark 8. The algorithm described above are explicit. A symbolic PROMPT-LTL model checking algorithm follows from the translation of PROMPT-LTL to the $\mu$-calculus described later in Theorem 14 The translation, however, involves a significant blow up. A symbolic algorithm that performs well on the colored Büchi graphs is left open. For standard Büchi graphs, algorithms can be classified as ones that are based on a nested fixed point that calculates the set of states that can reach $\alpha$ infinitely often [8], and ones that calculate symbolically the MSCC of the graph [5]. We believe that algorithms of the second type can be extended to colored graphs. 


\section{Assume-Guarantee Model Checking}

For two systems $\mathcal{S}=\left\langle A P, S, \rho, s_{0}, L\right\rangle$ and $\mathcal{S}^{\prime}=\left\langle A P, S^{\prime}, \rho^{\prime}, s_{0}^{\prime}, L^{\prime}\right\rangle$, the parallel composition of $\mathcal{S}$ with $\mathcal{S}^{\prime}$, denoted $\mathcal{S} \| \mathcal{S}^{\prime}$, is the system that contains all the joint behaviors of $\mathcal{S}$ and $\mathcal{S}^{\prime}$. Formally, $\mathcal{S} \| \mathcal{S}^{\prime}=\left\langle A P, S^{\prime \prime}, \rho^{\prime \prime}, s_{0}^{\prime \prime}, L^{\prime \prime}\right\rangle$, where $S^{\prime \prime} \subseteq S \times S^{\prime}$ contains exactly all pairs that agree on their label, that is $\left\langle s, s^{\prime}\right\rangle \in S^{\prime \prime}$ iff $L(s)=L^{\prime}\left(s^{\prime}\right)$. Then, $s_{0}^{\prime \prime}=$ $\left\langle s_{0}, s_{0}^{\prime}\right\rangle$ and $\rho^{\prime \prime}\left(\left\langle s, s^{\prime}\right\rangle,\left\langle t, t^{\prime}\right\rangle\right)$ iff $\rho(s, t)$ and $\rho^{\prime}\left(s^{\prime}, t^{\prime}\right)$. Finally, $L^{\prime \prime}\left(\left\langle s, s^{\prime}\right\rangle\right)=L(s)$.

An assume-guarantee specification for a system $\mathcal{S}$ is a pair of two specifications $\varphi_{1}$ and $\varphi_{2}$. The system $\mathcal{S}$ satisfies the specification, denoted $\left\langle\varphi_{1}\right\rangle \mathcal{S}\left\langle\varphi_{2}\right\rangle$, if it is the case that for all systems $\mathcal{S}^{\prime}$, if $\mathcal{S} \| \mathcal{S}^{\prime}$ satisfies $\varphi_{1}$, then $\mathcal{S} \| \mathcal{S}^{\prime}$ also satisfies $\varphi_{2}$ [12]. In the context of LTL it is not hard to see that $\left\langle\varphi_{1}\right\rangle \mathcal{S}\left\langle\varphi_{2}\right\rangle$ iff $\mathcal{S} \models \varphi_{1} \rightarrow \varphi_{2}$. Intuitively, since the \| operator amounts to taking the intersection of the languages of $\mathcal{S}$ and $\mathcal{S}^{\prime}$, it is sound to restrict attention to systems $\mathcal{S}^{\prime}$ that correspond to single computations of $\mathcal{S}$. In the case of PROMPT-LTL, we can also restrict attention to single computations, but we have to take the bounds into an account. Formally, we have the following.

Lemma 2. Consider a system $\mathcal{S}$ and PROMPT-LTL formulas $\varphi_{1}$ and $\varphi_{2}$. The specification $\left\langle\varphi_{1}\right\rangle \mathcal{S}\left\langle\varphi_{2}\right\rangle$ does not hold iff there is a bound $k_{1} \geq 0$ such that for every bound $k_{2} \geq 0$, there is a trace $w$ of $\mathcal{S}$ such that $\left(w, 0, k_{1}\right) \models \varphi_{1}$ but $\left(w, 0, k_{2}\right) \not \models \varphi_{2}$.

Since refuting assume-guarantee specifications refer to two bounds, we extend the alternating-color technique to refer to two sets of colors. The atomic proposition $p$ partitions the computation to blocks that bound $k_{1}$, and a new atomic proposition $q$ does the same for $k_{2}$. According to Lemmas 1 and 2 refuting $\left\langle\varphi_{1}\right\rangle \mathcal{S}\left\langle\varphi_{2}\right\rangle$ amounts to finding a bound $k_{1} \geq 0$ such that for all bounds $k_{2} \geq 0$, there is a computation $w$ of $\mathcal{S}$ such that $w$ has a $k_{1}$-bounded $p$-coloring that satisfies alt $t_{p} \wedge \operatorname{rel}_{p}\left(\varphi_{1}\right)$, but $w$ also has a $k_{2}$-spaced $q$-coloring that satisfies $a l t_{q} \wedge \neg \operatorname{rel}_{q}\left(\varphi_{2}\right)$. Indeed, such a computation satisfies $\varphi_{1}$ with bound $k_{1}$, and does not satisfy $\varphi_{2}$ with bound $k_{2}$.

We now show that the pumpable nonemptiness technique developed in Section 4 for solving the model-checking problem can be used also for solving the assume-guarantee model-checking problem, only that now the corresponding colored Büchi graphs are colored with two sets of colors, one for $\varphi_{1}$ and one for $\varphi_{2}$. Also, the definition of when a path in the graph is pumpable corresponds to the intuition above.

A colored Büchi graph of degree two is a tuple $G=\left\langle\{p, q\}, V, E, v_{0}, L, \alpha\right\rangle$. It is similar to a colored Büchi graph, only that now there are two sets of colors, described by $p$ and $q$. Accordingly, $L: V \rightarrow 2^{\{p, q\}}$. Also, $\alpha$ is a generalized Büchi condition of index 2, thus $\alpha=\left\{\alpha_{1}, \alpha_{2}\right\}$. A path $\pi=v_{0}, v_{1}, v_{2}, \ldots$ of $G$ is pumpable if we can pump all its $q$-blocks without pumping its $p$-blocks. Formally, if $i$ and $i^{\prime}$ are adjacent $q$-change points, then there are positions $j, j^{\prime}$, and $j^{\prime \prime}$ such that $i \leq j<j^{\prime}<j^{\prime \prime}<i^{\prime}$, $v_{j}=v_{j^{\prime \prime}}$ and $p \in L\left(v_{j}\right)$ iff $p \notin L\left(v_{j^{\prime}}\right)$. Also, $\pi$ is fair if it visits both $\alpha_{1}$ and $\alpha_{2}$ infinitely often. The pumpable nonemptiness problem is to decide, given $G$, whether it has a pumpable fair path.

Let $c\left(\varphi_{1}\right)=\operatorname{alt}_{p} \wedge \operatorname{rel}_{p}\left(\varphi_{1}\right)$ and $\bar{c}\left(\varphi_{2}\right)=\operatorname{alt}_{q} \wedge \neg \operatorname{rel}_{q}\left(\varphi_{2}\right)$, and let $\mathcal{A}_{c\left(\varphi_{1}\right)}=$ $\left\langle 2^{A P \cup\{p\}}, Q_{1}, \delta_{1}, q_{0}^{1}, \alpha_{1}\right\rangle$, and $\mathcal{A}_{\bar{c}\left(\varphi_{2}\right)}=\left\langle 2^{A P \cup\{q\}}, Q_{2}, \delta_{2}, q_{0}^{2}, \alpha_{2}\right\rangle$ be the corresponding NBWs (per Theorem 3). We define the product $\mathcal{P}$ of $\mathcal{S}$ with $\mathcal{A}_{c\left(\varphi_{1}\right)}$ and $\mathcal{A}_{\bar{c}\left(\varphi_{2}\right)}$ as the colored Büchi graph of degree two. Thus, $\mathcal{P}=\left\langle\{p, q\}, S \times 2^{\{p, q\}} \times Q_{1} \times\right.$ $\left.Q_{2}, M,\left\langle s_{0},\{p, q\}, q_{0}^{1}, q_{0}^{2}\right\rangle, L,\left\{S \times 2^{\{p, q\}} \times \alpha_{1} \times Q_{2}, S \times 2^{\{p, q\}} \times Q_{1} \times \alpha_{2}\right\}\right\rangle$, where 
$M\left(\left\langle s, c, q_{1}, q_{2}\right\rangle,\left\langle s^{\prime}, c^{\prime}, q_{1}^{\prime}, q_{2}^{\prime}\right\rangle\right)$ iff $\rho\left(s, s^{\prime}\right), q_{1}^{\prime} \in \delta_{1}\left(q_{1}, L(s) \cup(c \cap\{p\})\right)$, and $q_{2}^{\prime} \in$ $\delta_{2}\left(q_{2}, L(s) \cup(c \cap\{q\})\right)$. Finally, $L\left(\left\langle s, c, q_{1}, q_{2}\right\rangle\right)=c$.

Theorem 9. The specification $\left\langle\varphi_{1}\right\rangle \mathcal{S}\left\langle\varphi_{2}\right\rangle$ does not hold iff the product of $\mathcal{S}$ with $\mathcal{A}_{c\left(\varphi_{1}\right)}$ and $\mathcal{A}_{\bar{c}\left(\varphi_{2}\right)}$ is pumpable nonempty,

As detailed in the full version, solving the nonemptiness of colored Büchi graphs of degree two requires a slight modification of the algorithms in Section5, we have to add the requirement that every $q$-block includes more than one $p$-block. The complexities stay the same, NLOGSPACE-complete and in linear time. This, together with Theorems 3 and 9 , imply the upper bound in the following theorem. The lower bound follows from the known lower bound for LTL.

Theorem 10. The assume-guarantee model-checking problem for PROMPT-LTL is PSPACE-complete and can be solved in time exponential in the length of the formulas and linear in the size of the system.

Remark 11. For LTL, fairness constraints about the system can be specified in the formula. Thus, checking that $\varphi_{2}$ holds in all computations that satisfy the fairness constraint $\varphi_{1}$ can be reduced to model checking $\varphi_{1} \rightarrow \varphi_{2}$. A fairness assumption can also be specified in PROMPT-LTL. Here, however, one has to allow the fairness assumption and the specification to be satisfied with different bounds. Thus, fairness should be reduced to checking $\left\langle\varphi_{1}\right\rangle \mathcal{S}\left\langle\varphi_{2}\right\rangle$.

For two formulas $\varphi_{1}$ and $\varphi_{2}$, we say that $\varphi_{1}$ implies $\varphi_{2}$ iff for every system $\mathcal{S}$, if $\mathcal{S}$ satisfies $\varphi_{1}$, then it also satisfies $\varphi_{2}$. In the case of LTL, $\varphi_{1}$ implies $\varphi_{2}$ iff the formula $\varphi_{1} \rightarrow \varphi_{2}$ is valid. In the case of PROMPT-LTL, $\varphi_{1}$ implies $\varphi_{2}$ iff $\left\langle\varphi_{1}\right\rangle \mathcal{U}\left\langle\varphi_{2}\right\rangle$, where $\mathcal{U}$ is the universal system (a clique over $2^{A P}$ that contains all traces over $A P$ ). Indeed, since for every system $\mathcal{S}$ we have that $\mathcal{S} \| \mathcal{U}=\mathcal{S}$, then $\left\langle\varphi_{1}\right\rangle \mathcal{U}\left\langle\varphi_{2}\right\rangle$ does not hold iff there is a system $\mathcal{S}$ such that if $\mathcal{S}$ satisfies $\varphi_{1}$ but $\mathcal{S} \not \models \varphi_{2}$. Since $\mathcal{U}$ is exponential in $A P$, and the PSPACE complexity of assume-guarantee model checking originates from an algorithm that is polynomial in the formulas and only logarithmic in the system, we have the following (the lower bound follows from the PSPACE hardness of LTL implication).

Theorem 12. The implication problem for PROMPT-LTL is PSPACE-complete.

\section{Expressiveness}

In this section we study expressiveness aspects of PROMPT-LTL. We show that a PROMPT-LTL formula $\varphi$ has an equivalent LTL formula iff $\varphi$ and live $(\varphi)$ are equivalent, thus the problem of deciding whether $\varphi$ can be translated to LTL is PSPACEcomplete. Since the semantics of PROMPT-LTL is defined with respect to a system, a natural question is whether we can translate PROMPT-LTL formulas to branching temporal logics. We show that indeed, all PROMPT-LTL formulas can be translated to the $\mu$-calculus.

All our results refer to finite-state systems. Thus, we say that two formulas $\varphi$ and $\varphi^{\prime}$ are equivalent iff for all finite systems $\mathcal{S}$, we have that $\mathcal{S} \models \varphi$ iff $\mathcal{S} \models \varphi^{\prime}$. 
Some PROMPT-LTL formulas $\varphi$ are equivalent to the LTL formula live $(\varphi)$. For example, it is not hard to see that $\mathbf{F}_{\mathbf{p}} r$ is equivalent to $\mathbf{F} r$, for an atomic proposition $r$. On the other hand, as demonstrated in Section 1 the PROMPT-LTL formula $\mathbf{F}_{\mathbf{p}} \mathbf{G} r$ is not equivalent to the LTL formula $\mathbf{F G} r$. Is $\mathbf{F}_{\mathbf{p}} \mathbf{G} q$ equivalent to another LTL formula? A negative answer follows from the fact that for every PROMPT-LTL formula $\varphi$, there is some LTL formula equivalent to $\varphi$ iff $\varphi$ is equivalent to live $(\varphi)$. Since the implication live $(\varphi) \rightarrow \varphi$ can be checked in PSPACE (the other direction is always valid), we have the following. The lower bound is proven by a reduction from LTL satisfiability.

Theorem 13. Deciding whether a PROMPT-LTL formula has an equivalent LTL formula is PSPACE-complete.

It is not hard to prove that the PROMPT-LTL formula $\mathbf{F}_{\mathbf{p}} \mathbf{G} q$ is equivalent to the CTL formula AFAG $q$. Indeed, a system satisfies both formulas iff there is a bound $k \geq 0$ such that all the computations may visit a state in which $q$ does not hold only in the first $k$ positions. One may wonder whether this argument can be generalized, leading to a simple translation of PROMPT-LTL formulas to CTL * formulas: given a PROMPT-LTL formula $\varphi$, translate it to a $\mathrm{CTL}^{\star}$ formula $\varphi^{\prime}$ by (recursively) replacing all subformulas of the form $\mathbf{F}_{\mathbf{p}} \theta$ by $\mathbf{F A} \theta$ (and adding an external $\mathbf{A}$ ). To see that the reduction does not hold in general, consider the PROMPT-LTL formula $\varphi=\mathbf{F}_{\mathbf{p}}(\mathbf{X} q \vee \mathbf{G} q)$. While the system $\mathcal{S}$ from Figure 1 satisfies $\varphi$ (with bound 3), the system $\mathcal{S}$ does not satisfy the $\mathrm{CTL}^{\star}$ formula $\varphi^{\prime}=\mathbf{A F A}(\mathbf{X} q \vee \mathbf{G} q)$. The question whether PROMPT-LTL can be expressed in $\mathrm{CTL}^{\star}$ is open. On the other hand, the two-color technique can be used in order to translate a PROMPT-LTL formula over $P$ to alternating parity tree automaton over the alphabet $2^{P \cup\{p\}}$, and then to the $\mu$-calculus over $P$. Formally, we have the following.

Theorem 14. Every PROMPT-LTL formula has an equivalent $\mu$-calculus formula of exponential length.

Acknowledgment. We thank the reviewers for many helpful comments.

\section{References}

1. Alur, R., Etessami, K., Torre, S.L., Peled, D.: Parametric temporal logic for model measuring. ACM ToCL 2(3), 388-407 (2001)

2. Alpern, B., Schneider, F.B.: Defining liveness. IPL 21, 181-185 (1985)

3. Biere, A., Artho, C., Schuppan, V.: Liveness checking as safety checking. In: Proc. 7th FMICS, ENTCS, vol. 66(2) (2002)

4. Beer, I., Ben-David, S., Geist, D., Gewirtzman, R., Yoeli, M.: Methodology and system for practical formal verification of reactive hardware. In: Dill, D.L. (ed.) CAV 1994. LNCS, vol. 818, pp. 182-193. Springer, Heidelberg (1994)

5. Bloem, R., Gabow, H.N., Somenzi, F.: An algorithm for strongly connected component analysis in $n \log n$ symbolic steps. In: Johnson, S.D., Hunt, Jr., W.A. (eds.) FMCAD 2000. LNCS, vol. 1954, pp. 37-54. Springer, Heidelberg (2000)

6. Büchi, J.R., Landweber, L.HG.: Solving sequential conditions by finite-state strategies. Trans. AMS 138, 295-311 (1969)

7. Eisner, C., Fisman, D.: A Practical Introduction to PSL. Springer, Heidelberg (2006) 
8. Emerson, E.A., Lei, C.-L.: Efficient model checking in fragments of the propositional $\mu$ calculus. In: Proc. 1st LICS, pp. 267-278 (1986)

9. Emerson, E.A., Mok, A.K., Sistla, A.P., Srinivasan, J.: Quantitative temporal reasoning. In: Clarke, E., Kurshan, R.P. (eds.) CAV 1990. LNCS, vol. 531, pp. 136-145. Springer, Heidelberg (1991)

10. Manna, Z., Pnueli, A.: The Temporal Logic of Reactive and Concurrent Systems: Specification. Springer, Berlin (1992)

11. Pnueli, A.: The temporal logic of programs. In: Proc. 18th FOCS, pp. $46-57$ (1977)

12. Pnueli, A.: In: Transition from global to modular temporal reasoning about programs. Logics and Models of Concurrent Systems, vol. F-13 of NATO Advanced Summer Institutes, pp. 123-144 (1985)

13. Pnueli, A., Rosner, R.: On the synthesis of a reactive module. In: Proc. 16th POPL, pp. 179190 (1989)

14. Tarjan, R.E.: Depth first search and linear graph algorithms. SIAM Journal of Computing 1(2), 146-160 (1972)

15. Vardi, M.Y.: Automata-theoretic model checking revisited. In: Cook, B., Podelski, A. (eds.) VMCAI 2007. LNCS, vol. 4349, pp. 137-150. Springer, Heidelberg (2007)

16. Vardi, M.Y., Wolper, P.: An automata-theoretic approach to automatic program verification. In: Proc. 1st LICS, pp. 332-344 (1986)

17. Vardi, M.Y., Wolper, P.: Reasoning about infinite computations. I\&C 115(1), 1-37 (1994) 